\title{
Investigation of abnormal absorption of cosmic-ray hadrons in lead calorimeters
}

\author{
A.S. Borisov ${ }^{1, *}$, V.G. Denisova ${ }^{1}$, V.I. Galkin ${ }^{2}$, Z.M. Guseva ${ }^{1}$, E.A. Kanevskaya ${ }^{1}$, \\ M.G. Kogan ${ }^{1}$, R.A. Mukhamedshin ${ }^{3}$, S.I. Nazarov ${ }^{2}$, V.S. Puchkov ${ }^{1}$, and T.Kh.Sadykov ${ }^{4}$ \\ ${ }^{1}$ P.N.Lebedev Physical Institute of the Russian Academy of Sciences, Moscow, Russia \\ ${ }^{2}$ Institute of Nuclear Physics, M.V.Lomonosov Moscow State University, Moscow, Russia \\ ${ }^{3}$ Institute for Nuclear Research of the Russian Academy of Sciences, Moscow, Russia \\ ${ }^{4}$ Institute of Physics and Technology, Almaty, Republic of Kazakhstan
}

\begin{abstract}
A phenomenon of abnormally weak absorption of very high energy cosmic ray hadrons in thick lead blocks is discussed. This phenomenon was first observed in a Tien Shan high altitude experiment to study hadronic cores of extensive air showers (EAS) with a deep ionization calorimeter and encouraged researchers to introduce the hypothesis of the so-called long-flying or penetrating cosmic ray component. A similar effect was detected later with deep uniform lead X-ray emulsion chambers (XREC) at the Pamirs, which we discuss in detail. To establish the nature of the phenomenon we are have carried out dedicated experiments at the Tien Shan and at the Pamirs by exposing two-storey XRECs with large air gaps. According to detailed simulation of the chamber response, these experiments are very sensitive to the production of charmed hadrons in the forward kinematic region and can prove a hypothesis that the phenomenon under study can be explained on the assumption of very high values of charm particle production cross section at $\left\langle E_{\text {Lab }}\right\rangle \sim 75 \mathrm{TeV}$ in the forward kinematic region at $x_{\mathrm{Lab}} \gtrsim 0.1$ which are near the upper limit of recent results of collider experiments, i.e., $\sigma_{p p \rightarrow c \bar{c}} \sim 8 \mathrm{mb}$. The same factor makes it possible to fit all the features of the experimental hadron absorption curve observed by means of deep uniform lead XRECs, including its bending at a depth of $\sim 70$ c.u.
\end{abstract}

\section{Introduction}

A reduction of absorption rate of high energy (tens of $\mathrm{TeV}$ ) hadron cascades while passing the lead material was observed for the first time in 1973 - 1974 by V.Yakovlev et al. [1] at the Tien Shan High Mountain Research Station (TSS) of the P.N. Lebedev Physical Institute in an extensive air shower (EAS) experiment with so-called Big Ionization Calorimeter (BIC) of $36 \mathrm{~m}^{2}$ in area. The BIC contained lead absorber of $850 \mathrm{~g} / \mathrm{cm}^{2}$ thick which is five mean free paths for protons in lead [2] (see also BIC's scheme in Fig. 1 in Ref. [3]). Due to the big thickness of the calorimeter, this unique experiment makes it possible to study the hadronic component of EAS in details. According to the obtained experimental data, the attenuation length, $L\left(E_{h}\right)$, of the hadronic component of EAS cores increases with energy, $E_{h}$, released by hadronic cores in the calorimeter (see experimental data in Fig. 2 in Ref. [3]). To explain the effect, a hypothesis of the existence of the so-called long-flying cosmic-ray component was introduced [2]. After the discovery of charmed particles and when their parameters had been specified, I.Dremin et al. [4] showed that these experimental data could be explained assuming that the charmed-particle production cross section attains $\sim 30 \%$ of the inelastic proton-nucleus cross section (see also simulation results in Fig. 2 in Ref. [3]).

\footnotetext{
*e-mail: asborisov55@mail.ru
}

In addition, these particles could carry away a significant fraction of the primary particle energy.

An abnormally weak absorption of hadrons was also observed [5] in the Pamir experiment while exposing deep uniform lead XRECs $110 \mathrm{~cm}$ thick (see its scheme in Fig. 3 in Ref. [3])). In the range of $t<78$ radiation lengths, the absorption curve obeys the conventional exponential law with index $\lambda_{a b s}^{(1)}=212 \pm 19 \mathrm{~g} / \mathrm{cm}^{2}$. However, at larger depths, $t>78$ r.l., the absorption length of hadrons in lead changes and becomes as high as $\lambda_{a b s}^{(2)}=310 \pm 36 \mathrm{~g} / \mathrm{cm}^{2}$ (see Fig. 4 in Ref. [3]).

It was suggested $[6,7]$ that both phenomena, i.e., excess ionization in the hadron calorimeter and hadron excess in the deep uniform XREC, result from high values of the cross section of leading charm particle (D mesons and $\Lambda_{c}^{+}$hyperons) production at energies $E_{L a b} \sim 50 \mathrm{TeV}$. To attain a good fit of the experimental data with simulation calculations, it was necessary to assume that the forward-cone charm-production cross section rapidly increases with energy and amounts to $\sigma_{c \bar{c}}^{N N} \simeq 2-4 \mathrm{mb}$ at $E_{h} \gtrsim 20 \mathrm{TeV}$ in the forward kinematic region $\mathrm{x}_{L a b} \gtrsim 0.1$. Note, that in the early 90 -ies the measured values of charm production cross section were as small as tens of $\mu \mathrm{b}$ at then attainable accelerator energies $(\sqrt{s}=20-50 \mathrm{GeV})$. It means that charm production cross section should rapidly (almost linearly) increase with energy contradicting theoretical approaches available at that time. 


\section{Exposure of the two-storey XREC at the Tien Shan Mountains}

To prove this hypothesis, we carried out a dedicated experiment at the TSS located at an attitude of $3340 \mathrm{~m}$ above sea level $\left(700 \mathrm{~g} / \mathrm{cm}^{2}\right)$. This experiment employed a twostorey XREC with a large air gap between two vertically separated lead blocks of the chamber (see Fig. 5 in Ref. [3])).

The main idea of the experiment is to let charmed particles decay effectively within a gap of width of $H \simeq c \tau \gamma=$ $c \tau \cdot E / m \approx 2.5 \mathrm{~m}$ emitting $e^{ \pm}$particles and $\gamma$-rays within leptonic and semileptonic decay modes. Here $\tau$ is the lifetime of D mesons, $\gamma, E$ and $m$ are their Lorentz factor, energy and mass, respectively. The emitted electromagnetic particles will generate electromagnetic cascades in the lower lead block and thus will manifest themselves as a bump on the absorption curve (details are shown in Fig. 6 in Ref. [3]) while high energy electromagnetic particles produced by cosmic rays above the chamber will be effectively screened out by the upper lead block. The magnitude of the bump should obviously correlate with the value of the charm production cross section.

To construct a two-storey XREC with a large air gap between two lead blocks at the TSS, a two storey laboratory building which has a "window" in the floor slabs between the storeys was used. The XREC was assembled at the top of ionization calorimeter (IC) installed at the ground floor just under the window. The final design of the two-storey XREC, exposed at the TSS in 2007 2008 , consisted of the upper and lower blocks with areas of $48 \mathrm{~m}^{2}$ and $32 \mathrm{~m}^{2}$, respectively, and 2.16-m air gap between them (see Fig. 7 in Ref. [3]). The lead plates of the both blocks were interleaved with X-ray films of RT-6F type manufactured in Russia. The X-ray films were used as sensitive elements of the chamber.

The upper block of the chamber consisted of a socalled $\Gamma$-block containing three layers of $X$-ray films under $3.5,5.0$ and $6.5 \mathrm{~cm}$ of lead and a conventional Pb-block which were separated by two layers of ionization chambers and a $1.5 \mathrm{~cm}$ thick lead absorber between them. The Pb-block has 23 lead layers. The thickness of the first lead layer is $2 \mathrm{~cm}$, while other lead layers are $1 \mathrm{~cm}$ thick each. In the central part of the Pb-block, the lead layers are interleaved with X-ray films covering only $12 \mathrm{~m}^{2}$ of its total area. Thus the total depth of the lead absorber in the upper block is $32 \mathrm{~cm}$. The lower block of the two-storey XREC includes 13 layers of X-ray films. The first one is placed under $3.5 \mathrm{~cm}$ of lead; the next six X-ray film layers are separated by $1 \mathrm{~cm}$ of lead, and the last six film layers are separated by $1.5 \mathrm{~cm}$ of lead.

The electromagnetic component of cosmic rays incident on the XREC is almost completely absorbed in the $\Gamma$-block. Taking into account the additional lead layer 1.5 $\mathrm{cm}$ thick and spacing effects due to the presence of two layers of rubber supports (3 and $6 \mathrm{~cm}$ thick each) as well as two layers of ionization chambers with thin copper casing, darkness spots observed in the $23 \mathrm{X}$-ray film layers of the $\mathrm{Pb}$-block can only be produced by cosmic ray hadrons in their interactions with lead. Thus the hadron-induced showers are the only source of darkness spots observed in the Pb-block. The estimated threshold energy of the recorded hadron-induced cascades is $\sim 6-8 \mathrm{TeV}$.

Due to the large 2.16-m air gap between the two blocks of the two-storey XREC the hadron-induced showers originate in the upper block practically vanish in the gap. Only penetrating hadrons and successive hadronic interactions in the lead absorber of the lower block can produce dark spots on X-ray films placed in the lower block. Therefore, we should observe a drastic decrease of the darkness spot intensity in the first six centimeters of the lower block if it were not for the charm production. Charm particles produced in the upper block effectively decay in the air gap, partially, with emitting electromagnetic particles which, in their turn, should generate electromagnetic showers in the lead absorber of the lower block.

This work presents the analysis of experimental data on the absorption of cosmic ray hadrons obtained during one year (2007 - 2008) exposure of a two-storey XREC with large air gap at the TSS. The experimental data are compared with simulations assuming various values of charm production cross section. The same comparison is performed for experimental data of the Pamir experiment with a deep (110-cm thick) uniform lead XREC.

\section{Simulation of the experiment and the XREC response}

Monte-Carlo simulations of both experiments, i.e., with the two-storey XREC and deep homogeneous lead XREC, were carried out under the assumption that incident cosmic ray hadrons at mountain altitudes are mainly represented by nucleons and pions with energies $E_{h} \geq 20 \mathrm{TeV}$ which are produced by protons and nuclei of the primary cosmic ray radiation in the thick target $\left(700 \mathrm{~g} / \mathrm{cm}^{2}\right)$ of the atmosphere above the chamber. It was assumed that relative fractions of incident nucleons and pions are $60 \%$ and $40 \%$ while indices of the power energy spectra for nucleons and pions are -3.10 and -3.22 , respectively.

Nuclear-electromagnetic cascades produced by incident nucleons and pions in XREC of both types were simulated with the software package ECSim 2.0 [9]. The ECSim 2.0 code is based on the GEANT 3.21 package and allows to calculate the detector response for XREC of a given design taking into account the exact experimental technique employed in the Pamir experiment including processing and measurement of darkness spots produced by electromagnetic cascades on X-ray films.

To generate nucleon-lead and pion-lead interactions accounting for the production of charm hadrons and their subsequent decay in the air gap with emission of $e^{ \pm}$and $\gamma$-rays, the FANSY1.0 model [10] was used which was incorporated in the ECSim 2.0 package. The FANSY1.0 Monte Carlo generator [10] represents a phenomenological hadronic interaction model based on quark-gluon string theoretical approaches and assumes charm particle production with various cross sections. In many features it is close to the QGSGET II model. 


\section{Comparison of experimental and simulated data}

While analyzing the results of the experiments on absorption of hadrons in XREC by comparing the experimental distributions with simulated ones, we faced the problem of a shortage of both experimental and simulated statistics due to the small exposure of the two-storey XREC at the TSS as well as to insufficient performance of the available computers. To make results of the experiment more robust to experimental errors and fluctuations in the development of nuclear-electromagnetic cascades we treated not only reconstructed individual cascades but also separate darkness spots as we observe them at each film layer, i.e., quite independently from layer to layer. This procedure increases the statistics of experimental data being analyzed and strongly diminishes the experimental ambiguities related to a rather complicated procedure of reconstructing cascades recorded with multi-layered XREC.

The comparison of Tien Shan experimental data with simulated hadron absorption curves calculated with ECSim2.0@FANSY1.0 code for the two-storey XREC is presented in Fig. 1. As for the simulated data, the darkness spot distributions by the depth of observation layers, $t$, in the chamber were obtained at optical density threshold for darkness spot observation $D_{\min }=0.40$, determined using aperture $R=84 \mu \mathrm{m}$, and were normalized to one Xray film and to one incident particle. Besides, we used three cases of charm production parameters which determine the following values of charm production cross section $\sigma_{h-P b \rightarrow c \bar{c}}^{p r o d}$ in the kinematic forward-cone or so-called fragmentation region, i.e., at $x_{L a b}>0.1:(1) \sigma_{h-P b \rightarrow c \bar{c}}^{p r o d} \sim 0$ (green squares), (2) $\sim 6 \mathrm{mb} /$ nucleon (red circles), (3) $\sim 8$ $\mathrm{mb} /$ nucleon (brown stars).

In the case of negligible charm production cross section $\sigma_{h-P b \rightarrow c \bar{c}}^{p r o d} \sim 0$, there is a drastic fall of the spot number in the initial layers of X-ray films in the lower block of the two-storey XREC just after the bottom of the upper block (or after the layer corresponding to $32 \mathrm{~cm}$ of lead absorber in the plotted distribution). Then we observe a gradual restoration of distribution points to the same exponential dependence as observed in the upper block of the XREC. Such a behavior of the darkness spot distribution is explained with a lead-air-lead transition effect taking into account the large width of the air gap and specific features of hadron cascades in lead absorbers which are governed by a large magnitude of the nuclear interaction length $\left(\lambda_{\text {int }}^{\pi-P b} \approx 10.5 \mathrm{~cm}\right)$ and a short radiation length in lead $\left(X_{0} \approx 0.56 \mathrm{~cm}\right)$.

However, if the charm production cross section is as high as $\sigma_{h-P b \rightarrow c \bar{c}}^{\text {prod }} \sim 6-8 \mathrm{mb} /$ nucleon, we observe a bump on the darkness spot distribution at the initial layers of X-ray films in the lower block due to the appearance of electromagnetic showers generated via the decay of charmed particles (such as $\mathrm{D}^{ \pm}, \mathrm{D}^{0}, \mathrm{D}^{*}$ mesons, $\Lambda_{c}$ hyperons) in the air gap. The relative amplitude of this bump increases with increasing $\sigma_{h-P b \rightarrow c \bar{c}}^{p r o d}$ that makes it possible to measure the value of the charm production cross section at $x_{\text {Lab }} \gtrsim 0.1$ in experiments with two-storey XRECs.

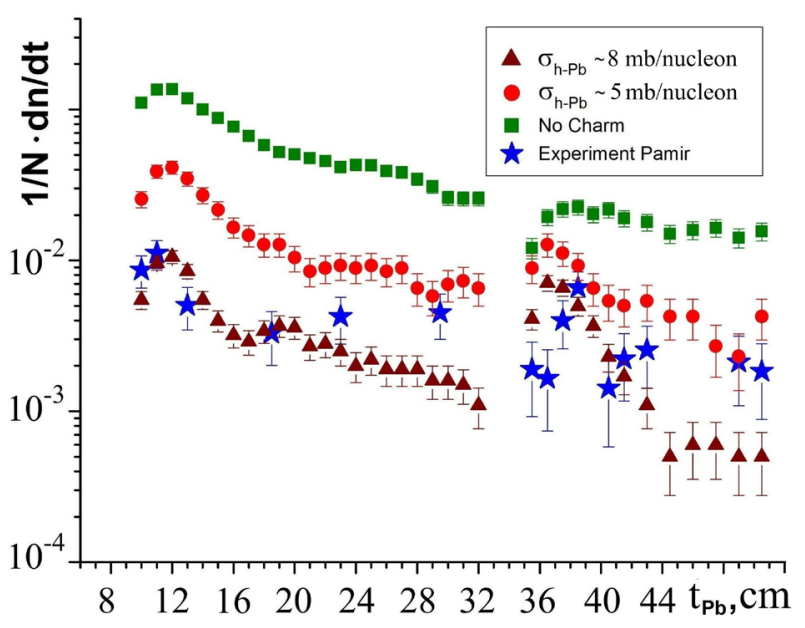

Figure 1. Darkness spot distribution, normalized to an X-Ray film, by the depth, $t$, of observation layers in the chamber expressed in centimeters in the Tien Shan experiment with twostorey XREC: experimental data (blue stars) and three sets of simulated data with three different charm production cross sections, i.e., $\sigma_{h-P b \rightarrow c \bar{c}}^{p r o d} \sim 8,6$ and $0 \mathrm{mb} /$ nucleon at optical density threshold $D_{\min }=0.40$ (in the case of simulations only). The fractions of nucleons and pions among incident particles are assumed to be $60 \%$ and $40 \%$, respectively, and angular distributions of incident hadrons are taken into account.

The experimental data on dark spots observed in the Tien Shan two-storey XREC are also plotted in Fig. 1.

To compare the experimental data with simulated ones adequately, the experimental distribution was normalized to the simulated one calculated with $\sigma_{h-P b \rightarrow c \bar{c}}^{p r o d} \sim 6$ $\mathrm{mb} /$ nucleon at the point corresponding to the observation layer under $10 \mathrm{~cm}$ of lead in the upper block of the twostorey XREC.

Note that simulated distributions were calculated accounting for measured angular distributions of incident hadrons and on the assumption that the observation threshold of the dark spots on X-ray films is $D_{\min }=0.40$, where $D$ is the optical density of the spot measured by photometers with diaphragm radius $R=84 \mu \mathrm{m}$. However, we applied a different procedure for experimental spot selection in order to increase the experimental statistics, i.e., we selected dark spots, first, by naked eyes and then distinguished the true spots of electromagnetic origin with the optical magnifier. This difference in selection criteria resulted in some difference between the experimental and simulated distributions of dark spots.

One can see from Fig. 1 that the simulated distribution corresponding to case No. 3 fits the experimental data rather well unless you consider some shift in the position of the experimental bump to the larger observation depths compared with simulated distributions.

We believe that this shift in the position of two peaks of electromagnetic origin is due to the above mentioned difference in dark spot selection criteria. To prove this, we analyzed the dependence of electromagnetic shower maxima in lead absorbers on energy with different dark spot 


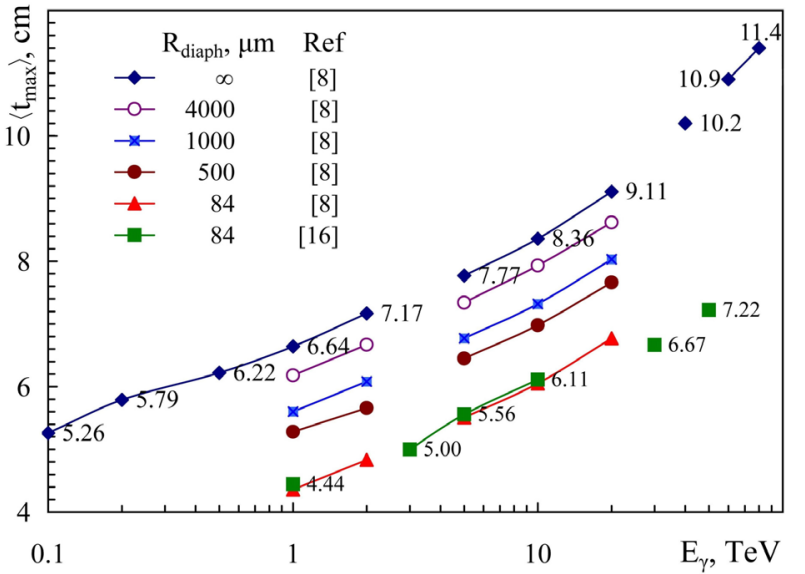

Figure 2. Depth of electron-photon cascade-development maximum $\left\langle t_{\max }\right\rangle$ in lead as a function of energy of the incident $\gamma$-ray depending on the size of the apperture applied for counting em particles with $E_{t h r}=100 \mathrm{keV}$. The LPM effect is taken into account.

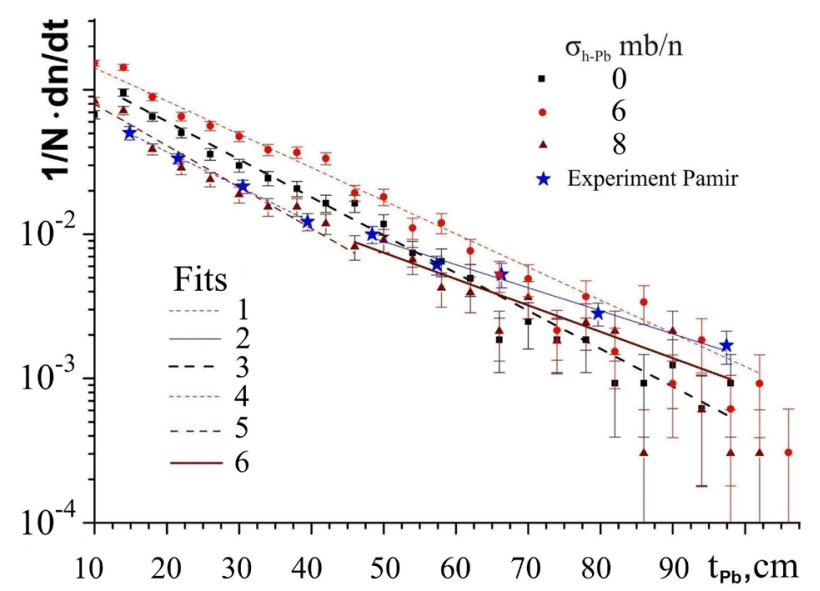

Figure 3. Distributions of hadron cascade origin points produced in homogeneous lead XREC. Experimental points are shown by stars. Simulated data are obtained for $\sigma_{h-P b \rightarrow c \bar{c}}^{\text {prod }} \sim 8$ (triangles), 6 (circles) and $0 \mathrm{mb} /$ nucleon at $x_{\text {Lab }} \gtrsim 0.1$ on the assumption that the fractions of nucleons and pions among incident particles are $60 \%$ and $40 \%$, respectively.

selection, namely: a) maxima are determined accounting for all e-m particles with $E_{t h r}=100 \mathrm{keV}$; b) maxima are determined accounting for e-m particles within radius of the diaphragm $R=84 \mu \mathrm{m}, 500 \mu \mathrm{m}, 1000 \mu \mathrm{m}, 4000 \mu \mathrm{m}$ at the same $E_{t h r}=100 \mathrm{keV}$. Case a) is close to our experimental selection procedure with the naked eye. The results of the analysis is presented in Fig. 2. One can see that the average maxima of e-m cascade development, $\left\langle t_{\text {max }}\right\rangle$, measured accounting for all cascade particles are more than $2 \mathrm{~cm}$ deeper than those determined by counting shower particles within $R=84 \mu \mathrm{m}$. Therefore the shift in the position of electromagnetic cascades in the experiment as compared to our calculation results seems rather reasonable.
Table 1. Values of index $\Lambda_{a b s}$ in $\exp \left(-t / \Lambda_{a b s}\right)$ fits (see Fig. 3.)

\begin{tabular}{cllll}
\hline $\begin{array}{c}\text { Fit } \\
\text { number }\end{array}$ & $\begin{array}{l}\sigma_{c \bar{c}}^{\text {prod }}, \\
\mathrm{mb}\end{array}$ & $\begin{array}{l}t \text { range, } \\
\mathrm{cm}\end{array}$ & $\begin{array}{l}\Lambda_{a b s}, \\
\mathrm{~g} / \mathrm{cm}^{2}\end{array}$ & $\begin{array}{l}\Lambda_{a b s}, \\
\mathrm{~cm}\end{array}$ \\
\hline 1 & Exprt. & $<45$ & $202 \pm 8$ & $17.8 \pm 0.7$ \\
2 & Exprt. & $<45$ & $307 \pm 24$ & $27.1 \pm 2.1$ \\
3 & 0 & $10-110$ & $188 \pm 6$ & $16.5 \pm 0.5$ \\
4 & 6 & $10-110$ & $214 \pm 9$ & $18.9 \pm 0.8$ \\
5 & 8 & $<45$ & $171 \pm 11$ & $15.1 \pm 1.0$ \\
6 & 8 & $>45$ & $269 \pm 32$ & $23.7 \pm 2.8$ \\
\hline
\end{tabular}

The same approach for explaining the abnormal behavior of hadron penetration in lead was applied to the analysis of the experimental data obtained with deep uniform lead XRECs 110 -cm thick which were exposed at the Pamirs in the 80-ies [5]. The experimental distribution of the cascade origin points for hadrons with $E_{h}^{(\gamma)} \gtrsim 6.3 \mathrm{TeV}$ obtained in these XRECs is presented in Fig. 3.

The experimental data are compared with simulated ones calculated with ECSim2.0@FANSY 1.0 code assumping three different values of charm-production cross section, namely, $\sigma_{h-P b \rightarrow c \bar{c}}^{\text {prod }} \sim 0,6$ and $8 \mathrm{mb} /$ nucleon at $x_{\text {Lab }}>0.1$. As follows from this figure, the data set, simulated with $\sigma_{h-P b \rightarrow c \bar{c}}^{\text {prod }} \sim 8 \mathrm{mb} /$ nucleon, fits the experimental data well enough. It even becomes possible to reproduce the bending of the hadron absorption curve at the depths of $t \sim 45 \mathrm{~cm}$. For lower values of the charm-production cross section ( $\sim 6 \mathrm{mb}$ /nucleon), the distribution of the origin points of hadron cascades can be approximated by a single exponential law for the whole absorber depth contradicting the experimental data.

Table 1 presents values of absorption lengths $\Lambda_{a b s}$ obtained by fitting the experimental and simulated distributions of hadron cascade origin points produced in homogeneous lead XREC with exponentials $\exp \left(-t / \Lambda_{a b s}\right)$ (see Fig. 3).

\section{Discussion}

The ALICE, LHCb and ATLAS collaborations have measured inclusive $d \sigma / d p_{t}$ cross sections of open-charm mesons in $p p$ collisions at $\sqrt{s}=2.76$ and $7 \mathrm{TeV}$. These results are very interesting from the theoretical point of view, especially results from pseudorapidity regions $2<\eta<4$ and $\eta<2.1$, obtained by LHCb and ATLAS, which can improve our understanding of $\mathrm{pQCD}$ production of heavy quarks (see, e.g., [11-13]).

Unfortunately, particles, produced in the most forward cone of phase space with high values of pseudorapidities $\eta$ (i.e., in the so-called fragmentation region of a projectile particle), are practically unobservable by collider experiments due to their specific constructional traits (finite dimension of the acceleration pipe which hinders any displacement of detectors close to the colliding axis). On the contrary, cosmic ray experiments with fixed target make it possible to investigate the fragmentation region of projectile particles, i.e., $x_{L a b} \gtrsim 0.1$, where the behavior of charm production with energy could be different from that observed in the central range. In any case, cosmic ray ex- 
periments could give some complementary information to collider data.

The significance of the forward region greatly increases with interaction energy, so that it begins to play a key role at the primary energies under investigation (for instance, at the LHC design energy $E_{0} \sim 10^{17} \mathrm{eV}$ almost $90 \%$ of the collision energy is released in the region with $\eta \geq 6$ ).

The first evidence of the rapid growth of charm hadroproduction cross sections with energy in the mid rapidity range was presented by the STAR/RHIC Collaboration [14]. Soon these results were confirmed by PHENIX Collaboration [15]. A strong energy dependence of the total charm-production cross section was revealed: $\sigma_{p p \rightarrow c \bar{c}} \propto$ $E^{0.8}$.

At $\sqrt{s}=2.76$ and $7 \mathrm{TeV}$, measurements carried out by the ALICE, ATLAS, CMS and LHCb Collaborations are in fair agreement [12] with each other:

$\sigma_{p p \rightarrow c \bar{c}}^{t o t}(2.76 \mathrm{TeV})=4.8 \pm 0.8$ (stat) ${ }_{-1.3}^{+1.0}($ syst. $) \mathrm{mb}$,

$\sigma_{p p \rightarrow c \bar{c}}^{t o t}(7 \mathrm{TeV})=8.5 \pm 0.5$ (stat) ${ }_{-2.4}^{+1.0}($ syst. $) \mathrm{mb}$.

To get the values of total charm production cross sections cited above, the measured cross sections were extrapolated to the full phase space by scaling the measured cross section by the ratio of the total cross section over the cross section in the experimentally covered phase space calculated using the NLO pQCD technique.

Taking into account rather large uncertainties of this procedure, one can conclude that cosmic ray experiments provide high energy physicists with complementary information on the charm hadroproduction mechanism.

As follows from the comparison of charm production cross sections obtained in accelerator experiments and in cosmic ray experiments, cosmic ray values seem to be too high. We believe that accounting for high energy incident muons (including so-called prompt muons originating via the decay of charm particles) and implementation of more hard spectra of secondary particles in the FANSY1.0 model in accordance with recent LHC results will decrease the value of charm production cross-section. A possible contribution of methodical errors into the experimental data must be analyzed in detail as well.

\section{Conclusion}

The analysis of experimental data obtained in two high altitude experiments with a two-storey XREC and a deep uniform XREC shows that an abnormally weak absorption of hadrons in the thick lead absorber can be explained by a rapid increase of the forward-cone charm production cross section $\sigma_{h-P b \rightarrow c \bar{c}}^{\text {prod }}$ with energy up to such high values as $6-8 \mathrm{mb} /$ nucleon at energies $\left\langle E_{h}^{\text {Lab }}\right\rangle \sim 75 \mathrm{TeV}$. However, the available experimental statistics does not allow us to exclude other possible hypotheses, for instance, the existence of some additional long-flying cosmic ray component like strangelets which can also contribute to the observed effects.

\section{References}

[1] V.S. Aseikin et al. Izvestiya of AN of USSR, ser. fiz. (1974) 5, 998 (in Russian)

[2] S.I. Nikolsky, E.L. Feinberg, V.P. Pavluchenko, V.I. Yakovlev. Preprint FIAN, N 69 (1975) (in Russian)

[3] A.S. Borisov et al. EPJ Web of Conferences 99, 10004 (2015)

[4] I.M. Dremin, V.I. Yakovlev. Topics on Cosmic Rays. 60th Anniv. C.M.G. Lattes. Campinas (1984) 1, 122

[5] I.V. Rakobolskaya al. Features of interactions of cosmic-ray hadrons of superhigh energies. MGU, Moscow (2000) (in Russian)

[6] V.I. Yakovlev. Proc. 24th ICRC, Roma (1995) 1, 446

[7] I.M. Dremin, D.T. Madigozhin, V.I. Yakovlev. Proc. 21st ICRC, Adelaida, 1990, V. 10, P. 166.

[8] L.G.Sveshnikova, O.P.Strogova. Proc. 23rd ICRC, Calgary (1993) v. 4, p. 33.

[9] A.S. Borisov, V.G. Denisova, V.I. Galkin et al. Proc. 30th ICRC, Merida (2007) v. 4, 593

[10] R.A.Mukhamedshin. Eur.Phys.J. C 60, 345 (2009)

[11] The ALICE Collaboration, J. High Energy Phys. 01 (2012) 128; The ALICE Collaboration. arXiv:1203.2160v4 [nucl-ex]

[12] The LHCb Collaboration, LHCb-CONF-2010-013

[13] The ATLAS Collaboration, ATLAS-CONF-2011017

[14] A. Tai, J. Phys. G 30 (2004) S809. The STAR collaboration. arXiv:1204.4244v3 [nucl-ex]

[15] S. Kelly, J. Phys. G 30 (2004) S1189

[16] A.A. Belyaev, I.Ivanenko et al. Electron-photon cascades in cosmic rays ar superhigh energies. Nauka, Moscow (1980) (in Russian) 